\title{
Anti-nociceptive, anti-inflammatory and toxicological evaluation of Fang-Ji-Huang-Qi-Tang in rodents
}

\author{
Yu-Chin Lin ${ }^{1}$, Ching-Wen Chang ${ }^{2,3}$ and Chi-Rei Wu ${ }^{3 *}$
}

\begin{abstract}
Background: Fang-Ji-Huang-Qi-Tang (abbreviated as FJHQT), composed by six medicinal herbs including Radix Stephania Tetrandra, Radix Astragali, Rhizoma Atractylodis Macrocephalae, Radix Glycyrrhizae, Rhizoma Zingiberis and Fructus Ziziphi Jujubae, is a frequently Chinese prescription for treating painful and inflammatory disorders such as rheumatoid arthritis. When Radix Stephania Tetrandra was misused with Aristolochia species, acute or chronic nephropathy caused by aristolochic acid was happened. Thus, the present study was aimed to identify Radix Stephania Tetrandra and performed the pharmacological and toxicological evaluation of FJHQT extract in rodents.

Methods: Radix Stephania Tetrandra was identified by macroscopic and microscopic observation, and the content of tetrandrine in FJHQT extract was measured by high performance liquid chromatography. Then, the pharmacological activities of FJHQT extract with respect to clinical use was investigated with acetic acid-induced writhing response, formalin-induced licking response and carrageenan-induced paw edema. Finally, we evaluated the subacute toxicology of FJHQT extract after 28-day repeated oral administration in rats.
\end{abstract}

Results: Radix Stephania Tetrandra was correctly used in FJHQT extract, and the content of tetrandrine in FJHQT extract was $2.5 \mathrm{mg} / \mathrm{g}$. FJHQT extract produced a pronounced and dose-dependent antinociceptive and anti-inflammatory effects in three above models. FJHQT extract after 28-day repeated administration did not caused any hematological, biochemical and histological change in rats.

Conclusions: We suggest that FJHQT extract is a high safety index Chinese medicine for antinociceptive and anti-inflammatory application when Radix Stephania Tetrandra was correctly used in FJHQT. Its antinociceptive and anti-inflammatory mechanism might be related to peripheral nociceptive pathway such as prostaglandins.

Keywords: Fang-Ji-Huang-Qi-Tang, Radix Stephania Tetrandra, Tetrandrine, Antinociceptive, Anti-inflammatory, Subacute toxicology

\section{Background}

Fang-Ji-Huang-Qi-Tang (abbreviated as FJHQT), a frequently Chinese remedy for relieving pain and edema from abdominal pain and rheumatoid arthritis, is composed of Radix Stephania Tetrandra (the roots of Stephania tetrandra), Radix Astragali (the roots of Astragalus membranaceus), Rhizoma Atractylodis Macrocephalae (the roots of Atractylodes macrocephala), Radix Glycyrrhizae (the roots of Glycyrrhiza uralensis), Rhizoma Zingiberis

\footnotetext{
* Correspondence: crw@mail.cmu.edu.tw

${ }^{3}$ Department of Chinese Pharmaceutical Sciences and Chinese Medicine Resources, College of Pharmacy, China Medical University, No. 91, Hsieh Shih Road, Taichung 40402, Taiwan

Full list of author information is available at the end of the article
}

(the roots of Zingiber officinale) and Fructus Ziziphi Jujubae (the fructus of Ziziphus jujuba) [1]. Pharmacological reports indicated that FJHQT possessed hepatoprotective, renal-protective, and immune-modulatory activities [1]. Many reports pointed out that Radix Stephaniae Tetrandrae and Radix Astragali possessed antiinflammatory activities in vitro and in vivo [2-6]. However, no scientific report regarding the antinociceptive and antiinflammatory activities of FJHQT has been published. Therefore, the present study was attempted to investigate the antinociceptive and anti-inflammatory effects of FJHQT extract in rodents with acetic acid-induced 
writhing test [7], formalin-induced licking test [8] and carrageenan-induced edema test [9].

The dry roots of Aristolochia plants are being used as an alternative for Radix Stephaniae Tetrandrae in China and European for a long time, but the former contains aristolochic acid to cause acute and chronic nephropathy. Therefore, the present study was measured the content of tetrandrine in FJHQT extract by high performance liquid chromatography (HPLC) and evaluated the subacute toxicology of FJHQT extract after 28-day repeated oral administration.

\section{Methods}

\section{Preparation of plant extract}

Radix Stephania Tetrandra (TWU-Plantec-FIJHQT-0001), Radix Astragali (TWU-Plantec-FIJHQT-0002), Rhizoma Atractylodis Macrocephalae (TWU-Plantec-FIJHQT-0003), Radix Glycyrrhizae (TWU-Plantec-FIJHQT-0004), Rhizoma Zingiberis (TWU-Plantec-FIJHQT-0005) and Fructus Ziziphi Jujubae (TWU-Plantec-FIJHQT-0006) were purchased from Taiwan market and identified with macroscopic and microscopic method by Professor Lin Y. C.. These characteristics include vascular bundles and the morphology and distribution of vascular bundles, catheters and fibers etc. These above medicinal materials were deposited in the Department of Biotechnology, TransWorld University. FJHQT (17.5 kg) was composed of these above medicinal materials at a ratio of 10:10:6:3:3:3. They were washed separately and put in the oven at $70^{\circ} \mathrm{C}$ to dry until its water content is below $5 \%$. Then they were cut into thin slices and put into a container $(100 \times 100 \times$ $100 \mathrm{~cm}^{3}$ ). Distilled water was added and heated at $100^{\circ} \mathrm{C}$ for 60 minutes consecutively. The solution was dried and concentrated with a rotary evaporator (Laborota 20 compact), purchased from Heidolph Instruments $\mathrm{GmbH} \& \mathrm{Co}$. (Schwabach, Germany), under $50^{\circ} \mathrm{C}$ and 120-180 mbar. The extract was grounded into powder, and sealed into a Pyrex glass bottle. The yield is about $32.8 \%$.

FJHQT extract $(25,50,100 \mathrm{mg} / \mathrm{kg})$ were dissolved in sterile distilled water and administered orally 60 minutes prior to the injection of an inducer. Control were received sterile distilled water in the same experiments. Indomethacin (INDO) $(10 \mathrm{mg} / \mathrm{kg})$ were prepared as suspension with $0.5 \%$ carboxymethylcellulose and administered intraperitoneally 30 minutes prior to the injection of an inducer.

\section{Subjects}

Male Sprague-Dawley rats (200-250 g) were used for the study of anti-inflammatory activities and toxicological evaluation. Male ICR mice (20-25 g) were used for testing the analgesic effects. The experimental protocol (Protocol No. 98-113-NH) was approved by the Institutional Animal Care and Use Committee (IACUC) of China Medical University and the care of animal was carried out according to the Guiding Principles for the Care and Use of Laboratory Animals. They were housed for at least 1 week before starting experiment in a temperature- $\left(23 \pm 1^{\circ} \mathrm{C}\right)$ and humidity- $(60 \%)$ regulated environment with free access to standard food in pellets and tap water, on a $12 \mathrm{~h}-12 \mathrm{~h}$ light/dark cycle (light phase: 08:00 to 20:00 h) was maintained. After 1 week of acclimatization, eight rodents each group in the below experiments were used. Then, the below drugs were administered, and the analgesic and antiinflammatory assays were operated by double-blind method. After behavioral measurement, all animals were killed by carbon dioxide.

\section{Measurement of tetrandrine in FJHQT by HPLC system}

The determination of tetrandrine from FJHQT extract was carried out by HPLC with a photodiode array detector. The HPLC system was consisted of a Shimadzu LC-20AT solvent delivery system, equipped with a SPDM20A photodiode array detector, set at $263 \mathrm{~nm}$. Samples were injected with SiL-20A autosampler to separate on the TSK-Gel ODS-100S column. All chromatographic operations were carried out at $25^{\circ} \mathrm{C}$. The mobile phase consisted of solvent A ( $0.3 \%$ formic acid) and solvent B (acetonitrile). The elution profile for $A$ was $0-10 \mathrm{~min}$, linear gradient change of $0-5 \%$; $10-40 \mathrm{~min}$, linear gradient change to 55\%; and maintained for another $10 \mathrm{~min}$ with a post run time in order to equilibrate the column and for the baseline to return to the normal and initial working conditions. Flow rate was $1.0 \mathrm{~mL} / \mathrm{min}$. FJHQT extract was dissolved in methanol and then filtered with a $0.22 \mu \mathrm{m}$ filter. Stock solutions of the standards were prepared in methanol to final concentrations of $1 \mathrm{mg} /$ $\mathrm{mL}$. Ten $\mu \mathrm{L}$ of standard or sample solutions was injected into the HPLC instrument for analysis in triplicate. The chromatographic peak of tetrandrine was confirmed by comparing their retention times and UV spectra.

\section{Acetic acid-induced abdominal writhing response in mice} Each mouse was given intraperitoneally $1 \%$ aqueous solution of acetic acid ( $10 \mathrm{~mL} / \mathrm{kg}$ body weight), and then was placed in the individual observation boxes. Five minutes after the injection of acetic acid, the number of writhing responses per mouse was counted for 10 minutes during acetic acid-induced abdominal writhing [7]. Finally, the number of writhing responses permitted us to express the percentage of protection using the following ratio: (Control mean - treated mean) $\times 100 /$ control mean .

\section{Formalin-induced licking response in mice}

This method represented a modification of that described by Shibata et al. [8]. Each mouse was placed in the observation chamber on an acrylic transparent plate floor for $5 \mathrm{~min}$ prior to the formalin injection. Beneath the floor, a large mirror was inclined at a $45^{\circ}$ angle in 
(A) Macroscopic

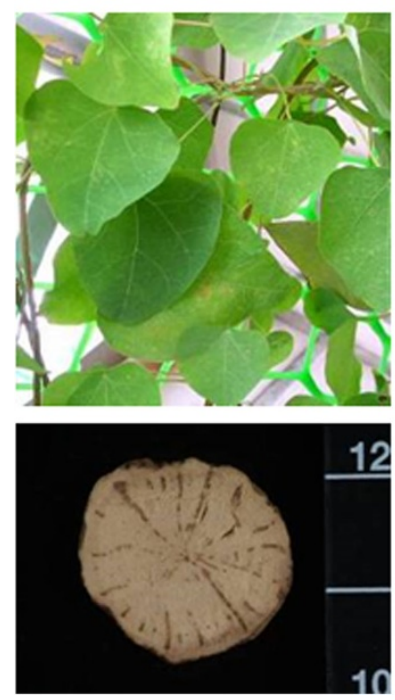

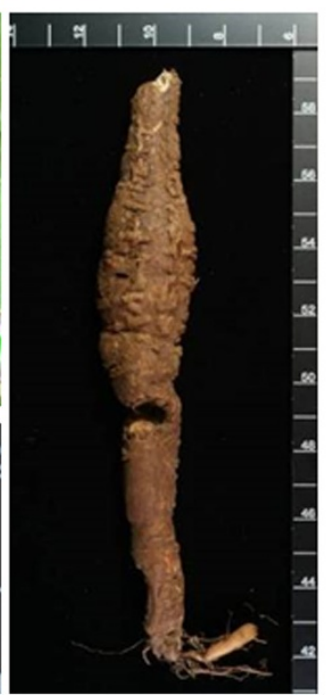

\section{(B) Microscopic}

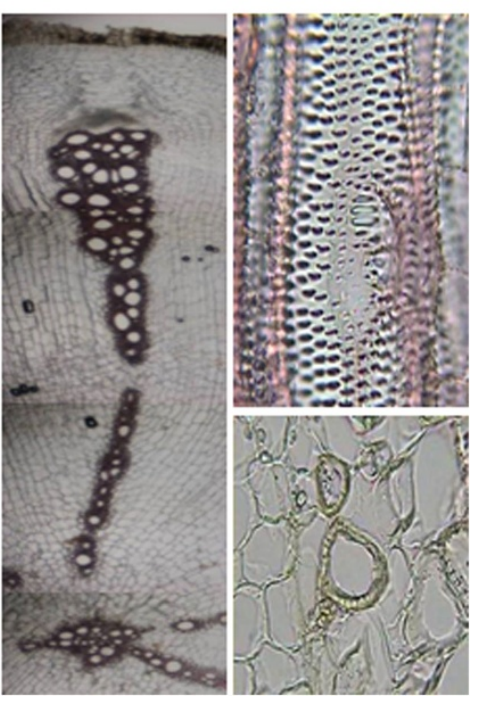

Figure 1 Pharmacognostic photographs of Radix Tetrandria. (A) Macroscopic characteristics (B) Microscopic characteristics.

order to allow clear observation of the paws of the animal. The animals were administered $25 \mu \mathrm{L}$ of $1 \%$ formalin into the right subplantar. Then, each animal was returned to the chamber and the two distinct periods of the intensive licking response was observed. The first period (early phase) was recorded 0-5 min after formalin injection and the second period (late phase) was recorded 10-35 min after formalin. The time (in seconds) spent in licking responses of the injected paw was measured as an indicator of pain response.

\section{Carrageenan-induced paw edema in rats}

The anti-inflammatory activity was determined in rats by measuring the mean increase in hind paw volume after the subplantar injection of carrageenan [9]. The animals were injected with $0.1 \mathrm{~mL} 1 \%$ carrageenan in the right hind foot under the plantar aponeurosis. The inflammation was quantitated in terms of milliliters using a plethysmometer (7150 Ugo Basile) which recorded small differences in water level caused by volume displacement. Before any treatment, the average volume of


Figure 2 HPLC chromatograms of aqueous extract of Fang-Ji-Huang-Qi-Tang at 263 nm. (A) Standard, (B) Fang-Ji-Huang-Qi-Tang. 
(A) Acetic acid-induced writhing response

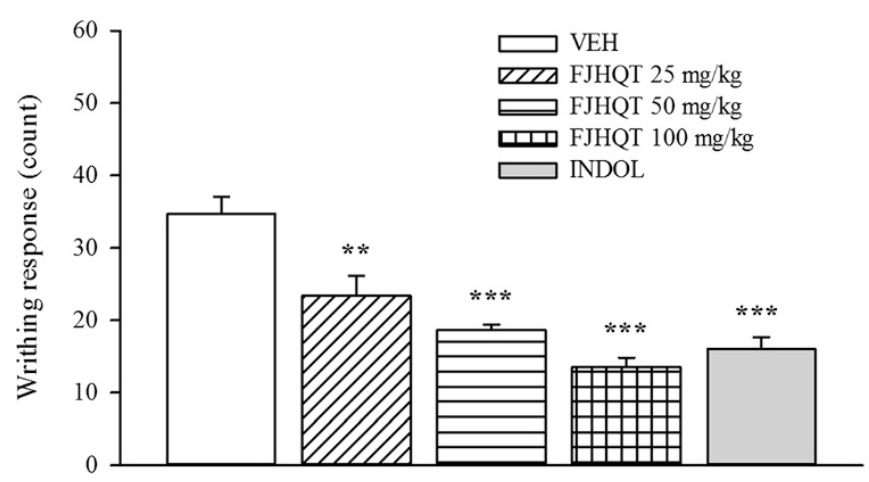

(B) Formalin-induced licking response

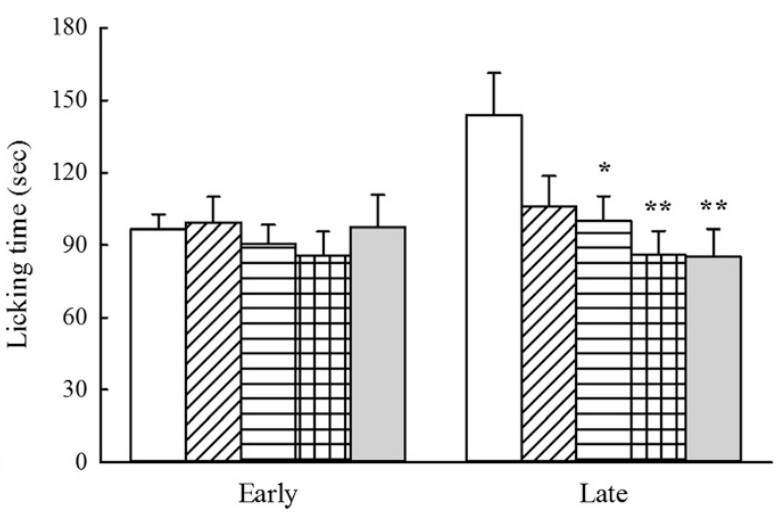

Figure 3 Effect of Fang-Ji-Huang-Qi-Tang extract (FJQHT, 25, 50 and $100 \mathrm{mg} / \mathrm{kg}$ ) and indomethacin (INDO, $10 \mathrm{mg} / \mathrm{kg}$ ) on (A) the acetic acid-induced writhing response, and $(B)$ the early (0-5 $\mathrm{min}$ ) and late phase (10-35 $\mathrm{min}$ ) of formalin-induced licking response in mice. Each values are represented as mean \pm S.E. $(N=8)$. ${ }^{*} P<0.05 .{ }^{* *} P<0.01 .{ }^{* * *} P<0.001$ as compared with the VEH group.

the backpaws of each animal was determined $\left(\mathrm{V}_{\mathrm{o}}\right)$, after 3 measurements which did not differ from more than $4 \%$ (preciseness of the apparatus). Then $30,60,90,120$, $150,180,210,240 \mathrm{~min}$ after carrageenan injection, the average volume of the backpaws of each animal was determined $\left(V_{t}\right)$, after 3 measurements which did not differ from more than $4 \%$. The percentages of edema at each record were calculated by compared the average volume of the backpaws of each animal $\left(\mathrm{V}_{\mathrm{t}}\right)$ after carrageenan injection with the average volume of the backpaws of each animal $\left(\mathrm{V}_{\mathrm{o}}\right)$ before any treatment. Percentages of inhibition were obtained for each group by using the following ratio: $\left[\left(\mathrm{V}_{\mathrm{t}}-\mathrm{V}_{\mathrm{o}}\right)\right.$ control $-\left(\mathrm{V}_{\mathrm{t}}-\mathrm{V}_{\mathrm{o}}\right)$ treated $] \times 100 /$ $\left(\mathrm{V}_{\mathrm{t}}-\mathrm{V}_{\mathrm{o}}\right)$ control.

\section{Subacute toxicity study in rats}

The 28-day repeated oral toxicity studies were carried out in rats according to the OECD test guideline 407 [10]. Rats were divided randomly into 4 groups of 8 animals each. After an overnight fast, control group which rats received sterile distilled water, whereas other groups which rats received FJHQT extract at the doses of 0.1, 0.5 , and $1.0 \mathrm{~g} / \mathrm{kg}$ body weight, respectively. Doses of FJHQT extract were administered daily by oral gavage in the volume of $10 \mathrm{~mL} / \mathrm{kg}$ body weight, once daily for 28 consecutive days. The rats were observed daily for any abnormal clinical signs and death during the study period. Body weight and food intake were measured and recorded daily during the study period. At the end of the study, all animals fasted overnight and, on 29th day, the animals were weighed. Blood was collected from retroorbital technique with or without EDTA for hematological and biochemical analysis, respectively. The animals were sacrificed and other body organs were taken out for detailed weight and histopathological changes.

\section{Hematological parameters and biochemical estimations}

Red blood cells (RBC), white blood cells (WBC), hematocrit (HCT), hemoglobin (HGB), mean corpuscular hemoglobin $(\mathrm{MCH})$, mean corpuscular hemoglobin concentration $(\mathrm{MCHC})$, mean corpuscular volume $(\mathrm{MCV})$, and platelet counts [11] were determined in control and FJHQT extract-treated groups. The serum was carefully aspirated into sample bottles for the various biochemical assays. Assay kits for aspartate transaminase (AST), alanine transaminase (ALT), creatinine, blood glucose,

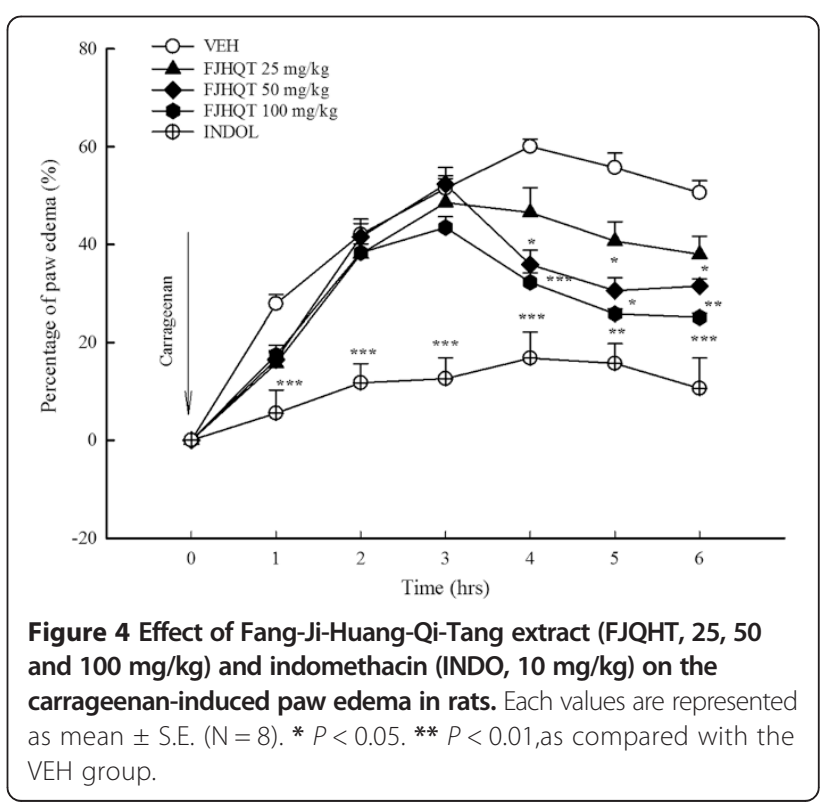




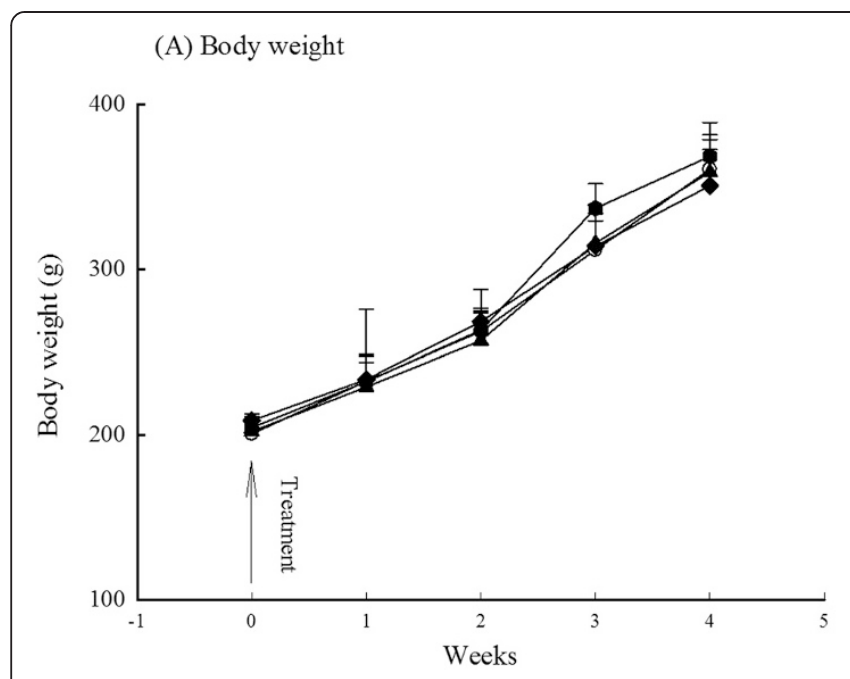

(B) Food intake

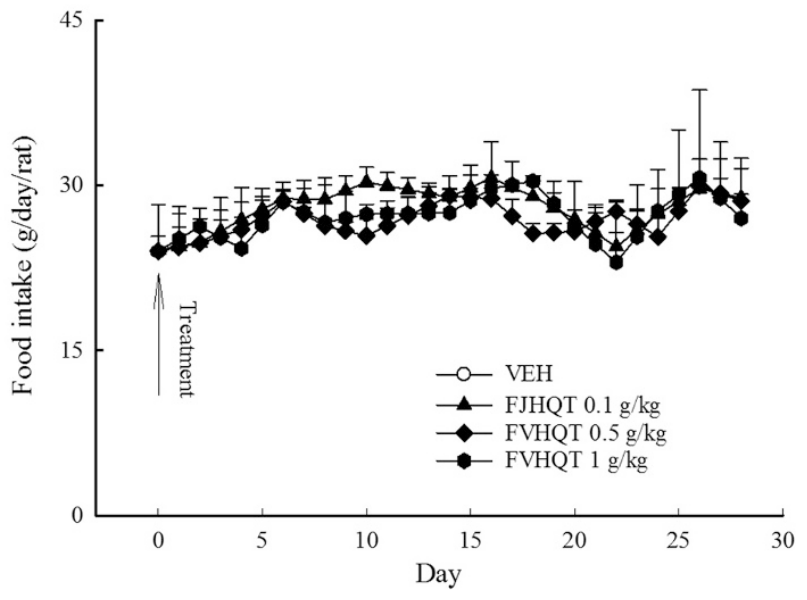

Figure 5 Effect of Fang-Ji-Huang-Qi-Tang extract (FJQHT, 0.1, 0.5 and $1.0 \mathrm{~g} / \mathrm{kg}$ ) on (A) the tendency of body weight and (B) daily food intake during 28-day repeated treatment in rats. Each value are represented as mean \pm S.E. $(N=8)$.

blood urea nitrogen (BUN), total protein and albumin analysis were purchased from Radox diagnostic kit and determined in the serum following the procedure described in the kits.

\section{Organs weight and histology}

The rats were quickly dissected and the brain, heart, lung, liver, spleen, kidney, adrenal, and testis were excised and weighed. The specimens for histopathology were fixed in $10 \%$ neutral, buffered formalin for $18 \mathrm{~h}$ at $4^{\circ} \mathrm{C}$. Thickness $(3-4 \mu \mathrm{m})$ of each specimen of liver and kidney was cut and stained with hematoxylin and eosin stain following the standard laboratory procedures. The stained sections were examined under microscope for any cellular damage or change in morphology of that particular tissue.

\section{Statistical analysis}

All data obtained during the antinociceptive and antiinflammatory activity was expressed in terms of mean and standard errors, and further analyzed by using ANOVA one-way analysis of variance, followed by Scheff's test. When probability $(P)$ was less than 0.05 , the difference was considered to be significant.

\section{Results and discussion}

Identification of Radix Stephania Tetrandra and measurement of tetrandrine content in FJHQT extract

Due to the misuse between Radix Stephania Tetrandra and Aristolochia species, we first identified Radix Stephania Tetrandra before the preparation of FJHQT extract with macroscopic and microscopic observation in medicinal material of Radix Stephania Tetrandra. The photographs of macroscopic characteristics including original plant and medicinal material of Radix Stephania Tetrandra were shown in Figure 1 (A). The oval or irregular stone cells in cortex and pits in round or oval reticulate vessels were found in the microscopic observation of Radix Stephania Tetrandra (Figure 1 (B)). These observed characteristics were same as the photographs of Radix Stephania Tetrandra in the literature [12]. The photographs of macroscopic and microscopic characteristics of other medicinal materials were shown in Additional file 1. We further quantified the content of tetrandrine in FJHQT extract after the preparation of FJHQT extract with HPLC. The chromatographs of tetrandrine and FJHQT extract were shown in Figure 2. Retention time of tetrandrine in HPLC chromatogram at $263 \mathrm{~nm}$ is about at $25.15 \mathrm{~min}$. The content of tetrandrine in FJHQT extract is

Table 1 Effects of Fang-Ji-Huang-Qi-Tang (FJQHT, 0.1, 0.5, $1.0 \mathrm{~g} / \mathrm{kg}$ ) after 28-day repeated oral administration on hematological parameters in rats

\begin{tabular}{|c|c|c|c|c|c|c|c|c|}
\hline Groups & $\operatorname{RBC}\left(10^{6}\right)$ & HGB (g/dL) & MCV (fj) & MCH (pg) & MCHC (g/dL) & HCT (\%) & WBC $\left(10^{3}\right)$ & Platelet $\left(10^{3}\right)$ \\
\hline Normal & $7.5 \pm 0.2$ & $14.5 \pm 0.2$ & $61.7 \pm 0.4$ & $19.4 \pm 0.1$ & $31.6 \pm 0.1$ & $45.9 \pm 0.7$ & $7.7 \pm 0.7$ & $1076.1 \pm 55.6$ \\
\hline FJHQT $0.1 \mathrm{~g} / \mathrm{kg}$ & $7.3 \pm 0.2$ & $15.0 \pm 0.4$ & $62.7 \pm 1.3$ & $20.1 \pm 0.3$ & $31.7 \pm 0.3$ & $44.8 \pm 0.8$ & $7.7 \pm 0.5$ & $1141.1 \pm 50.2$ \\
\hline FJHQT $0.5 \mathrm{~g} / \mathrm{kg}$ & $7.3 \pm 0.1$ & $14.5 \pm 0.2$ & $61.6 \pm 0.9$ & $20.0 \pm 0.4$ & $31.8 \pm 0.2$ & $47.9 \pm 1.2$ & $7.7 \pm 0.5$ & $1112.9 \pm 57.7$ \\
\hline FJHQT $1.0 \mathrm{~g} / \mathrm{kg}$ & $7.7 \pm 0.1$ & $14.8 \pm 0.2$ & $61.2 \pm 0.6$ & $19.3 \pm 0.2$ & $31.6 \pm 0.2$ & $46.9 \pm 0.7$ & $7.7 \pm 0.7$ & $1184.4 \pm 66.9$ \\
\hline
\end{tabular}

Data are represented with mean $\pm \mathrm{SEM}, \mathrm{N}=8$. 
Table 2 Effects of Fang-Ji-Huang-Qi-Tang (FJQHT, 0.1, 0.5, $1.0 \mathrm{~g} / \mathrm{kg}$ ) after 28-day repeated oral administration on plasma biochemical parameters in rats

\begin{tabular}{lllllllll}
\hline Groups & Glu $(\mathbf{m g} / \mathbf{d L})$ & TP $(\mathbf{m g} / \mathbf{d L})$ & Albumin $(\mathbf{m g} / \mathbf{d L})$ & Globulin $(\mathbf{m g} / \mathbf{d L})$ & ALT (U/L) & AST (U/L) & BUN (mg/dL) & Creatinine (mg/dL) \\
\hline Normal & $80.6 \pm 8.8$ & $7.2 \pm 0.9$ & $4.2 \pm 0.6$ & $3.0 \pm 0.3$ & $34.0 \pm 4.9$ & $12.9 \pm 1.8$ & $14.0 \pm 1.2$ & $0.8 \pm 0.1$ \\
FJHQT 0.1 g/kg & $85.4 \pm 8.3$ & $7.7 \pm 0.9$ & $4.5 \pm 0.6$ & $3.2 \pm 0.3$ & $32.1 \pm 3.2$ & $13.4 \pm 1.4$ & $13.5 \pm 1.7$ & $0.9 \pm 0.1$ \\
FJHQT 0.5 g/kg & $81.0 \pm 4.6$ & $7.5 \pm 0.3$ & $4.6 \pm 0.1$ & $2.9 \pm 0.2$ & $39.0 \pm 2.9$ & $14.8 \pm 1.1$ & $14.2 \pm 0.8$ & $0.7 \pm 0.2$ \\
FJHQT 1.0 g/kg & $82.4 \pm 3.0$ & $7.3 \pm 0.3$ & $4.4 \pm 0.2$ & $2.9 \pm 0.1$ & $38.1 \pm 1.0$ & $15.1 \pm 0.9$ & $13.8 \pm 1.0$ & $0.7 \pm 0.1$ \\
\hline
\end{tabular}

Data are represented with mean $\pm \mathrm{SEM}, \mathrm{N}=8$.

about $2.5 \pm 0.2 \mathrm{mg} / \mathrm{g}$ dry weight in accordance with the calibration curve of tetrandrine.

\section{Antinociceptive and anti-inflammatory activities of FJHQT extract}

Acetic acid-induced abdominal writhing response and formalin-induced licking response are very useful models for assessing the effects of antinociceptive drugs. To evaluate the antinociceptive activities of FJHQT extract, we performed the acetic acid-induced abdominal writhing response and formalin-induced licking response in mice. Acetic acid-induced abdominal writhing response is mainly based on the peripheral system, which involves prostaglandin synthesis via cyclooxygenase [13]. Our present result found pretreatment with FJHQT extract (25-100 mg/kg, po) decreased acetic acid-induced writhing response in a dose-dependent manner in mice (Figure $3(\mathrm{~A}))(P<0.01, P<0.001)$. Indomethacin, a positive control, at $10 \mathrm{mg} / \mathrm{kg}$ also decreased acetic acidinduced writhing response (Figure $3(\mathrm{~A}))(P<0.001)$. Thus, FJHQT extract possessed antinociceptive effect against the acetic acid-induced abdominal writhing response in mice. We further found that FJHQT extract (25-100 mg/kg, po) also significantly prevented the late but not early phase of formalin-induced licking response in mice (Figure 3(B)) $(P<0.05, P<0.01)$. Indomethacin also effectively inhibited the late but not early phase of formalin-induced licking response in mice (Figure $3(\mathrm{~B}))(P<0.01)$. In fact, some components of FJHQT extract such as Radix Stephania Tetrandra and Rhizoma Zingiberis have been proven to possess the antinociceptive activities in mice $[14,15]$. Moreover, recent report indicated that tetrandrine, an active ingredient of Radix Stephania Tetrandra, possessed the antinociceptive effect in mice [16]. Therefore, this result, confirmed the clinical use for the painful symptoms, showed that FJHQT extract produced pronounced and dose-related antinociceptive activities against acetic acid-induced writhing responses and the late phase of formalin-induced licking responses. Furthermore, there are obvious differential properties in the early and late phase of formalin-induced licking responses that the early phase is caused by central nerve fiber activation and the late phase is dependent on the functional changes in the peripheral nerves [8]. Thus, we suggested that the antinociceptive mechanism of FJHQT extract might be through the peripheral systems of pain pathway, in consistence with indomethacin.

Due to FJHQT extract only inhibited the late phase of formalin-induced licking response and early reports indicated the late phase seems to be an inflammatory response with inflammatory pain [8], we further investigated the anti-inflammatory effect of FJHQT extract with carrageenan-induced paw edema in rats. We found that pretreatment with FJHQT extract at 50-100 mg/kg inhibited carrageenan-induced edema formation from 4 to 6 hours after carrageenan injection in rats (Figure 4) $(P<0.05, P<0.01, P<0.001)$. Indomethacin at $10 \mathrm{mg} / \mathrm{kg}$, a positive control, also effectively inhibited carrageenaninduced edema formation throughout the measurement period in rats (Figure 4$)(P<0.001)$. Previous researchers have indicated that almost all components of FJHQT extract possessed anti-inflammatory activities in vitro and in vivo [17-22]. Tetrandrine also possessed antiinflammatory activities in vitro and in vivo [23-26]. Thus, this result, also confirmed the clinical use for the inflammatory disorders such as rheumatoid arthritis, showed that FJHQT extract also produced pronounced and dose-related anti-inflammatory activity against carrageenan-induced paw edema. All components of FJHQT extract produced a synergistic effect on the anti-inflammatory activity of FJHQT extract. Tetrandrine is also a major active ingredient of Radix Stephania Tetrandra and FJHQT extract.

Shibata et al. [8] suggested the early phase of formalininduced licking response was related to bradykinin and substance $\mathrm{P}$, and the late phase was related to bradykinin, autocirnes and prostaglandin. Moreover, previous researchers

Table 3 Effects of Fang-Ji-Huang-Qi-Tang (FJQHT, 0.1, 0.5, $1.0 \mathrm{~g} / \mathrm{kg}$ ) after 28-day repeated oral administration on urine parameters in rats

\begin{tabular}{lllll}
\hline Groups & Volumes $(\mathbf{m l})$ & $\mathbf{p H}$ value & $\begin{array}{l}\text { Protein } \\
(\mathbf{m g} / \mathbf{L})\end{array}$ & $\begin{array}{l}\text { Glucose } \\
(\mathbf{m m o l} / \mathbf{L})\end{array}$ \\
\hline Normal & $22.0 \pm 2.5$ & $6.8 \pm 0.1$ & $35.3 \pm 2.7$ & $6.8 \pm 1.5$ \\
FJHQT 0.1 g/kg & $21.4 \pm 2.5$ & $7.1 \pm 0.1$ & $39.9 \pm 3.5$ & $7.0 \pm 0.8$ \\
FJHQT 0.5 g/kg & $19.1 \pm 3.3$ & $7.0 \pm 0.3$ & $41.4 \pm 5.4$ & $6.9 \pm 1.0$ \\
FJHQT $1.0 \mathrm{~g} / \mathrm{kg}$ & $21.0 \pm 2.4$ & $7.1 \pm 0.1$ & $40.8 \pm 5.9$ & $6.7 \pm 0.7$ \\
\hline
\end{tabular}

Data are represented with mean $\pm \mathrm{SEM}, \mathrm{N}=8$. 
Table 4 Effects of Fang-Ji-Huang-Qi-Tang (FJQHT, 0.1, 0.5, $1.0 \mathrm{~g} / \mathrm{kg}$ ) after 28-day repeated oral administration on organ weight in rats

\begin{tabular}{lllllllll}
\hline Groups & Brain $(\mathbf{g})$ & Heart $(\mathbf{g})$ & Lung $(\mathbf{g})$ & Liver $(\mathbf{g})$ & Spleen $(\mathbf{g})$ & Kidney $(\mathbf{g})$ & Adrenal $(\mathbf{m g})$ & Testis $(\mathbf{g})$ \\
\hline Normal & $1.96 \pm 0.03$ & $1.25 \pm 0.05$ & $1.40 \pm 0.07$ & $9.37 \pm 0.44$ & $0.70 \pm 0.04$ & $2.50 \pm 0.08$ & $53 \pm 4$ & $2.91 \pm 0.03$ \\
FJHQT $0.1 \mathrm{~g} / \mathrm{kg}$ & $1.96 \pm 0.03$ & $1.30 \pm 0.04$ & $1.65 \pm 0.14$ & $9.24 \pm 0.24$ & $0.71 \pm 0.05$ & $2.52 \pm 0.06$ & $53 \pm 4$ & $3.08 \pm 0.16$ \\
FJHQT $0.5 \mathrm{~g} / \mathrm{kg}$ & $1.97 \pm 0.02$ & $1.21 \pm 0.04$ & $1.38 \pm 0.07$ & $9.02 \pm 0.45$ & $0.64 \pm 0.04$ & $2.43 \pm 0.11$ & $52 \pm 4$ & $2.91 \pm 0.03$ \\
FJHQT $1.0 \mathrm{~g} / \mathrm{kg}$ & $2.03 \pm 0.05$ & $1.32 \pm 0.04$ & $1.54 \pm 0.14$ & $9.45 \pm 0.22$ & $0.65 \pm 0.04$ & $2.55 \pm 0.08$ & $45 \pm 5$ & $3.18 \pm 0.12$ \\
\hline
\end{tabular}

Data are represented with mean $\pm \mathrm{SEM}, \mathrm{N}=8$.

have indicated that the mechanism of carrageenan-induced edema usually separates into three phases. First phase $(1.5 \mathrm{~h}$ after carrageenan treatment) is related to autocrines and PAF. Second phase (from $1.5 \mathrm{~h}$ to $2.5 \mathrm{~h}$ after carrageenan treatment) is related to kinins. Third phase $(2.5 \mathrm{~h}$ after carrageenan treatment) is related to prostaglandins and leukotrienes [27-29]. From our above results in three models, the antinociceptive and anti-inflammatory mechanism of FJHQT extract might be mainly related to bradykinin, autocirnes or prostaglandin pathway because FJHQT extract mainly inhibited the late-phase of formalin-induced licking response and the third phase of carrageenan-induced paw edema. Refereeing to the reports of all components of FJHQT extracts, their antiinflammatory mechanism might be mainly related to NF-kappaB, iNOS, cyclooxygenase-2 (COX-2) / prostaglandin (PGE) pathway [2,19,30,31]. Furthermore, some researchers indicated that tetrandrine also possessed the anti-inflammatory effect via the inhibition of NF-kappa / COX-2 pathway, the release of pro-inflammatory cytokines including IL-1 $\beta$, IL-6 and TNF- $\alpha$ in mice $[16,24,25,32]$. Therefore, our present results, in consistence with these literatures of all medicinal components and tetrandrine, demonstrated that the anti-inflammatory effects of FJHQT extract might be mainly through NF-kappaB, iNOS, cyclooxygenase-2 (COX-2)/prostaglandin (PGE) pathway, and then modulating the release of pro-inflammatory cytokines.

\section{Sub-acute toxicology of FJHQT extract}

Due to the misuse between Radix Stephania Tetrandra and Aristolochia species, Aristolochia species often cause acute or chronic nephropathy via aristolochic acid. Thus, we evaluated the subacute toxicology of FJHQT after 28-day oral administration in rats although we have identified the corrected use of Radix Stephania Tetrandra in FJHQT extract. Rats treated with FJHQT extract at $0.1,0.5$, or $1.0 \mathrm{~g} /$ $\mathrm{kg}$ body weight daily for 28 day, were survival and normal throughout the administration. FJHQT extract-treated rats did not show any changes in general behavior or other
(A)

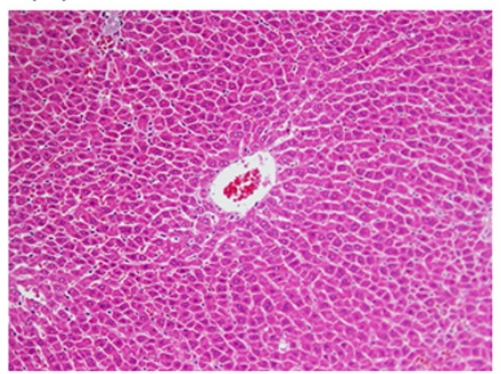

(C)

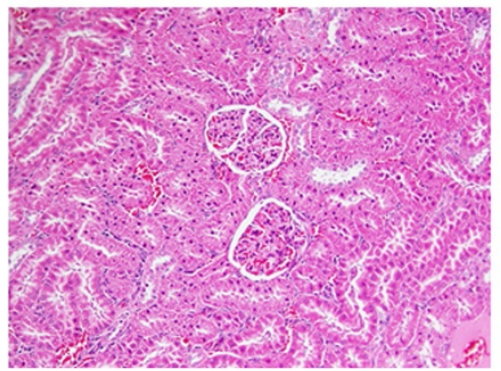

(B)



(D)

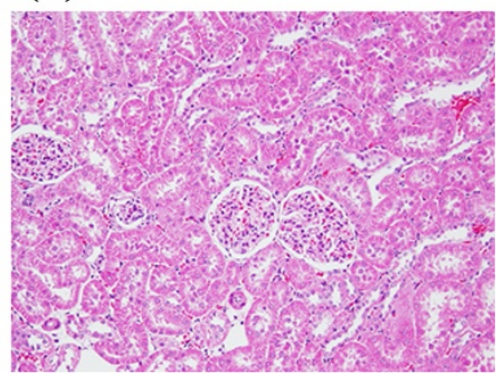

Figure 6 Histology of liver and kidney (H\&E stain, 100x) in rats. (A) and (C) Section of liver and kidney from vehicle-treated rats; (B) and (D) Section of liver and kidney from Fang-Ji-Huang-Qi-Tang (FJQHT, $1.0 \mathrm{~g} / \mathrm{kg}$ )-treated rats. 
physiological activities. No change was observed in body weight and food intake in FJHQT extract $(0.1,0.5$, or $1.0 \mathrm{~g} /$ $\mathrm{kg}$ )-treated groups compared with control group after 28 days-repeated treatment in rats (Figure 5(A) and (B)). Secondly, there were not significantly different between FJHQT extract $(0.1,0.5$, or $1.0 \mathrm{~g} / \mathrm{kg})$-treated rats and control rats in all hematological parameters including RBC, HGB, HCT, MCV, MCH, MCHC, WBC, and platelet counts (Table 1). Biochemical parameters for liver and kidney function test such as AST, ALT, creatinine, BUN, blood glucose, total protein, and albumin of FJHQT extract (0.1, 0.5 , or $1.0 \mathrm{~g} / \mathrm{kg}$ )-treated rats did not show any difference with those of control group (Table 2). The urine parameters including volume, $\mathrm{pH}$ value, protein and glucose of FJHQT extract $(0.1,0.5$, or $1.0 \mathrm{~g} / \mathrm{kg})$-treated rats did not show any difference with those of control group (Table 3). Finally, there were no difference between FJHQT extract (0.1, 0.5 , or $1.0 \mathrm{~g} / \mathrm{kg}$ )-treated rats and control rats in their mean weights and gross examinations of major organs including brain, heart, lung, liver, spleen, kidney, adrenal, and testis dissected from all rats (Table 4). When administered with higher dose of $1.0 \mathrm{~g}$ dose of FJHQT extract for 28 days, no histopathological changes in liver and kidney were observed (Figure 6). Thus, we suggested that FJHQT extract at 10 time effective dose for 28-day repeated oral administration did not cause any toxicological responses and histopathological changes if Radix Stephania Tetrandra in the component of FJHQT was not misused with Aristolochia species. Moreover, other report indicated tetrandrine at $50 \mathrm{mg} / \mathrm{kg}$ for 3-month repeated intraperitoneal administration caused death and moderate hydropic degeneration of the distal tubules in the kidneys in mice [33]. Thus, we elaborated that FJHQT extract has higher safety index in its antinociceptive and anti-inflammatory activities because the content of tetrandrine in the used dosage of FJHQT extract for the antinociceptive and anti-inflammatory tests is about $25-250 \mu \mathrm{g} / \mathrm{g}$ body weight (thousandth of chronic toxicological dose).

\section{Conclusion}

From our present results, FJHQT extract is a very safe and pronounced antinociceptive and anti-inflammatory Chinese prescription. These pharmacological activities of FJHQT extract confirms the clinical use for the painful symptoms caused by inflammatory disorders such as rheumatoid arthritis. The antinociceptive and anti-inflammatory activities of FJHQT extract were from the synergic effects of its medicinal components because many researchers have evidenced all medicinal components possessed antinociceptive and anti-inflammatory activities in vitro and in vivo [14,15,17-22], especially Radix Stephania Tetrandra. Furthermore, we reviewed the antinociceptive and antiinflammatory literatures of these medicinal components of FJHQT extract and found that their antinociceptive and anti-inflammatory mechanisms were due to the inhibition of NF-kappa B / COX-2 / iNOS pathway and the decrease of pro-inflammatory cytokines secretion such as IL-1 $\beta$ and TNF- $\alpha[2,19,30,31]$. Even the antinociceptive and antiinflammatory activities of tetrandrine also were through the inhibition of NF-kappa / COX-2 pathway, the release of pro-inflammatory cytokines including IL- $1 \beta$, IL- 6 and TNF$\alpha$ in mice $[16,24,25,32]$. Hence, we speculated the antinociceptive and anti-inflammatory mechanisms of FJHQT extract might be same as those of its medicinal components which be related to NF-kappa B / COX-2 / iNOS pathway and the release of pro-inflammatory cytokines, but this speculation should be investigated in the future.

\section{Additional file}

Additional file 1: Figure S1. Pharmacognostic photographs of Radix Astragali. (A) Macroscopic characteristics (B) Microscopic characteristics. Figure S2. Pharmacognostic photographs of Rhizoma Atractylodis Macrocephalae. (A) Macroscopic characteristics (B) Microscopic characteristics. Figure S3. Pharmacognostic photographs of Radix Glycyrrhizae. (A) Macroscopic characteristics (B) Microscopic

characteristics. Figure S4. Pharmacognostic photographs of Rhizoma Zingiberis. (A) Macroscopic characteristics (B) Microscopic characteristics. Figure S5. Pharmacognostic photographs of Fructus Ziziphi Jujubae. (A) Macroscopic characteristics (B) Microscopic characteristics.

\section{Abbreviations}

ALT: Alanine transaminase; AST: Aspartate transaminase; BUN: Blood urea nitrogen; FJHQT: Fang-Ji-Huang-Qi-Tang; HCT: Hematocrit; HGB: Hemoglobin; HPLC: High performance liquid chromatography; INDO: Indomethacin; MCH: Mean corpuscular hemoglobin; MCHC: Mean corpuscular hemoglobin concentration; MCV: Mean corpuscular volume; RBC: Red blood cells; WBC: White blood cells.

\section{Competing interests}

The authors declare that they have no competing interests.

\section{Authors' contributions}

CRW and YCL carried out all the experimentation, acquisition of data, conceived, designed, supervised the study, drafted and revised the manuscript. YCL and CWC identified the plants, and provided the plant extracts. All authors read and approved the final manuscript.

\section{Acknowledgments}

We thank the financial support of National Sciences Council CCMP98-CP-010, CCMP99-CP-010 and CCMP100-CP-010.

\section{Author details}

'Department of Biotechnology, TransWorld University, No.1221, Zhennan Rd., Yunlin County, Douliu City 64063, Taiwan. ${ }^{2}$ Department of Cosmetic Applications and Management, Mackay Junior College of Medicine, Nursing, and Management, No. 92, Shengjing Road, Beitou District, Taipei 11260, Taiwan. ${ }^{3}$ Department of Chinese Pharmaceutical Sciences and Chinese Medicine Resources, College of Pharmacy, China Medical University, No. 91, Hsieh Shih Road, Taichung 40402, Taiwan.

Received: 22 August 2014 Accepted: 15 January 2015

Published online: 05 February 2015

\section{References}

1. Wu YJ. Overview in the clinical and experimental research of Fang-JiHuang-Qi-Tang. Chinese Med Modern Distance Education of China. 2012;10:163-4. 
2. Li W, Sun YN, Yan XT, Yang SY, Kim S, Lee YM, et al. Flavonoids from Astragalus membranaceus and their inhibitory effects on LPS-stimulated pro-inflammatory cytokine production in bone marrow-derived dendritic cells. Arch Pharm Res. 2014;37:186-92.

3. Lai PK, Chan JY, Wu SB, Cheng L, Ho GK, Lau CP, et al. Anti-inflammatory activities of an active fraction isolated from the root of Astragalus membranaceus in RAW 264.7 macrophages. Phytother Res. 2014;28:395-404.

4. Ryu M, Kim EH, Chun M, Kang S, Shim B, Yu YB, et al. Astragali Radix elicits anti-inflammation via activation of MKP-1, concomitant with attenuation of p38 and Erk. J Ethnopharmacol. 2008;115:184-93.

5. Sekiya N, Shimada Y, Niizawa A, Kogure T, Mantani N, Sakai S, et al. Suppressive effects of Stephania tetrandra on the neutrophil function in patients with rheumatoid arthritis. Phytother Res. 2004;18:247-9.

6. Kang HS, Kim YH, Lee CS, Lee JJ, Choi I, Pyun KH. Anti-inflammatory effects of Stephania tetrandra S. Moore on interleukin-6 production and experimental inflammatory disease models. Mediators Inflamm. 1996;5:280-91.

7. Taber RI, Greenhouse DD, Rendell JK, Irwin S. Agonist and antagonist interactions of opioids on acetic acid-induced abdominal stretching in mice. J Pharmacol Exp Ther. 1969;169:29-38.

8. Shibata M, Ohkubo T, Takahashi H, Inoki R. Modified formalin test: characteristic biphasic pain response. Pain. 1989;38:347-52.

9. Winter CA, Risley EA, Nuss GW. Carrageenin-induced edema in hind paw of the rat as an assay for antiiflammatory drugs. Proc Soc Exp Biol Med. 1962;111:544-7.

10. OECD. Test No. 407: repeated Dose 28-day oral toxicity study in rodents. In: OECD Guidelines For the Testing of Chemicals, Section 4: Health Effects. OECD Publishing; 2008. http://www.oecd-ilibrary.org/environment/test-no407-repeated-dose-28-day-oral-toxicity-study-in-rodents_9789264070684-en

11. Wintrobe MM. Clinical Haemotology. Lea and Febigeer: Philadelphia, Pa, USA; 1961.

12. Pharmacopoeia Commission of the Ministry of Public Health. N0. 190: Fangji. In: A Coloured Altas of the Chinese Materia Medica Specified in Phamacopoeia of the People's Republic of China. Hong Kong: Joint Publishing (H.K.) Co. LTD; 1995.

13. Koo HJ, Lim KH, Jung HJ, Park EH. Anti-inflammatory evaluation of gardenia extract, geniposide and genipin. J Ethnopharmacol. 2006;103:496-500.

14. Li X, Zhang S, Qin L. Experimental study of analgesic effect of combined Radix Aconiti and Radix Stephaniae Tetrandrae. Zhongguo Zhong Xi Yi Jie He Za Zhi. 2000;20:202-4.

15. Ojewole JA. Analgesic, antiinflammatory and hypoglycaemic effects of ethanol extract of Zingiber officinale (Roscoe) rhizomes (Zingiberaceae) in mice and rats. Phytother Res. 2006;20:764-72.

16. Zhao H, Luo F, Li H, Zhang L, Yi Y, Wan J. Antinociceptive effect of tetrandrine on LPS-induced hyperalgesia via the inhibition of IKKbeta phosphorylation and the COX-2/PGE(2) pathway in mice. PLoS One. 2014;9:e94586.

17. Shen YC, Chou CJ, Chiou WF, Chen CF. Anti-inflammatory effects of the partially purified extract of radix Stephaniae tetrandrae: comparative studies of its active principles tetrandrine and fangchinoline on human polymorphonuclear leukocyte functions. Mol Pharmacol. 2001;60:1083-90.

18. Penna SC, Medeiros MV, Aimbire FS, Faria-Neto HC, Sertie JA, Lopes-Martins RA. Anti-inflammatory effect of the hydralcoholic extract of Zingiber officinale rhizomes on rat paw and skin edema. Phytomedicine. 2003;10:381-5.

19. Lee YS, Han OK, Park CW, Yang CH, Jeon TW, Yoo WK, et al. Pro-inflammatory cytokine gene expression and nitric oxide regulation of aqueous extracted Astragali radix in RAW 264.7 macrophage cells. J Ethnopharmacol. 2005;100:289-94.

20. Li CQ, He LC, Dong HY, Jin JQ. Screening for the anti-inflammatory activity of fractions and compounds from Atractylodes macrocephala koidz. J Ethnopharmacol. 2007;114:212-7

21. Goyal R, Sharma PL, Singh M. Possible attenuation of nitric oxide expression in anti-inflammatory effect of Ziziphus jujuba in rat. J Nat Med. 2011;65:514-8.

22. Yang XL, Liu D, Bian K, Zhang DD. Study on in vitro anti-inflammatory activity of total flavonoids from Glycyrrhizae Radix et Rhizoma and its ingredients. Zhongguo Zhong Yao Za Zhi. 2013;38:99-104.

23. Choi HS, Kim HS, Min KR, Kim Y, Lim HK, Chang YK, et al. Anti-inflammatory effects of fangchinoline and tetrandrine. J Ethnopharmacol. 2000;69:173-9.

24. Wu SJ, Ng LT. Tetrandrine inhibits proinflammatory cytokines, iNOS and COX-2 expression in human monocytic cells. Biol Pharm Bull. 2007;30:59-62.

25. Kang OH, An HJ, Kim SB, Mun SH, Seo YS, Joung DK, et al. Tetrandrine suppresses pro-inflammatory mediators in PMA plus A23187-induced HMC-1 cells. Int J Mol Med. 2014;33:1335-40.
26. Wang QS, Cui YL, Gao LN, Guo Y, Li RX, Zhang XZ. Reduction of the proinflammatory response by tetrandrine-loading poly(l-lactic acid) films in vitro and in vivo. J Biomed Mater Res A. 2014;102:4098-107.

27. Damas J, Bourdon V, Remacle-Volon G, Adam A. Kinins and peritoneal exudates induced by carrageenin and zymosan in rats. Br J Pharmacol. 1990;101:418-22.

28. Hwang SB, Lam MH, Li CL, Shen TY. Release of platelet activating factor and its involvement in the first phase of carrageenin-induced rat foot edema. Eur J Pharmacol. 1986;120:33-41.

29. Vinegar R, Schreiber W, Hugo R. Biphasic development of carrageenin edema in rats. J Pharmacol Exp Ther. 1969;166:96-103.

30. Chen J, Du CY, Lam KY, Zhang WL, Lam CT, Yan AL, et al. The Standardized Extract of Ziziphus jujuba Fruit (Jujube) Regulates Pro-inflammatory Cytokine Expression in Cultured Murine Macrophages: Suppression of Lipopolysaccharidestimulated NF-kappaB Activity. PTR: Phytotherapy research; 2014.

31. Jung HW, Yoon CH, Park KM, Han HS, Park YK. Hexane fraction of Zingiberis Rhizoma Crudus extract inhibits the production of nitric oxide and proinflammatory cytokines in LPS-stimulated BV2 microglial cells via the NF-kappaB pathway. Food Chem Toxicol. 2009;47:1190-7.

32. He FQ, Qiu BY, Li TK, Xie Q, de Cui J, Huang XL, et al. Tetrandrine suppresses amyloid-beta-induced inflammatory cytokines by inhibiting NF-kappaB pathway in murine BV2 microglial cells. Int Immunopharmacol. 2011;11:1220-5.

33. Yuan SY, Yang CR, Cheng CL, Hsu SL, Liao JW, Lin CC, et al. Comparative nephrotoxicity of aristolochic acid and tetrandrine in vitro and in vivo. Int J Toxicol. 2011;30:35-46.

\section{Submit your next manuscript to BioMed Central and take full advantage of:}

- Convenient online submission

- Thorough peer review

- No space constraints or color figure charges

- Immediate publication on acceptance

- Inclusion in PubMed, CAS, Scopus and Google Scholar

- Research which is freely available for redistribution 